\title{
Pensar la conectividad y la electronalidad en tiempos de la COVID-19: el WhatsApp y el modelo comunicativo del autismo
}

\author{
Thinking about connectivity and electronality in the days \\ of COVID-19: WhatsApp and the communicative model of \\ autism
}

(iD) Ernesto Reaño Carranza ${ }^{\circledR}$

Equipo de Investigación y Trabajo en Autismo (EITA). Lima, Perú

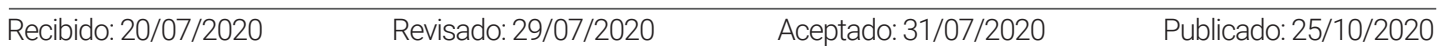

\section{RESUMEN}

La teoría de la electronalidad tal como ha sido vinculada con el autismo, establece que esta etapa de nuestra historia comunicacional, donde prima el uso de la palabra electrónica a través de las tecnologías nacidas de la cibernética y de la internet, ofrece herramientas que no sólo favorecen una mayor fluidez para un tipo de mente autista sino que benefician a la población en general. En estos tiempos de cuarentena producto de la COVID-19, ha sido necesario potenciar un mayor uso de herramientas y aplicaciones que favorecen la comunicación a distancia, uso para el cual hemos notado que las personas autistas parecen estar mejor preparadas debido a las formas de procesamiento que les son propias. Esto lleva a pensar en las relaciones entre la mente autista y las nuevas tecnologías de la información. Este artículo mostrará las relaciones entre esta y la configuración de los modelos comunicativos actuales centrados más en la conectividad que en el diálogo. Se propone, también una reflexión sobre lo que nos enseña la mente autista sobre las nuevas maneras de interactuar.

Palabras clave: Autismo, electronalidad, semiótica, WhatsApp, lingüística aplicada, conectividad, diálogo, COVID-19, habilidades sociales.

\begin{abstract}
The theory of electronality as it has been linked to autism, establishes that this stage of our communicational history, where the use of the electronic word prevails through technologies born from cybernetics and the internet, offers tools that not only favor greater fluidity for an autistic type of mind but benefit the general population. In these times of quarantine as a result of the COVID-19, it has been necessary to promote a greater use of tools and applications that favor remote communication, an use for which we have noticed that autistic people seem to be better prepared due to the forms of processing. This leads to thinking about the relationships between the autistic mind and the new information technologies. This article will show the relationships between the autistic mind and the configuration of current communication models focused more on connectivity than dialogue. It also proposes a reflection on what the autistic mind teaches us about new ways of interacting.
\end{abstract}


Keywords: Autism, electronality, semiotics, applied linguistics, connectivity, dialogue, COVID-19, social skills.

\section{INTRODUCCIÓN}

A partir del 15 de marzo del 2020, el Perú entró en una cuarentena producto de la pandemia desatada por la COVID-19. Uno de los sectores más afectados ha sido el educativo en todos sus niveles, cuyas clases tuvieron que ser adaptadas, en breve tiempo, a una modalidad a distancia y virtual, teniendo que asumir profesores y estudiantes el manejo y entendimiento de aplicaciones y programas no usados antes. Aún nos queda trabajo para graficar la totalidad los efectos que en la salud mental han provocado y provoca la situación de aislamiento y distancia física en la población general. En la actualidad la situación epidemiológica permite la reactivación de muchas de las actividades económicas que fueron paralizadas, la educación en los diferentes niveles, se ha propuesto que mantenga el formato a distancia durante este año, al menos.

La incertidumbre y el aislamiento han generado una serie de respuestas negativas en la población autista. El autismo en una condición del neurodesarrollo (Baron-Cohen, 2008) que se caracteriza por presentar un tipo de mente más preparada para sistematizar información y que encuentra dificultades en el aprendizaje de las habilidades sociales típicas necesarias para interactuar con el entorno. El tipo de mente autista requiere de altos grados de predictibilidad en su funcionamiento. Son personas, con una mente sistemática que busca constantes, por ello las rutinas, horarios y su cumplimiento son esenciales para su salud mental. La situación de cuarentena y el "nuevo orden", interpretado como "desorden" en la situación en casa, sin espacios para diferenciar los límites de la escuela con los del trabajo y las dificultades económicas, ha determinado un impacto profundo en las dinámicas familiares y particularmente en las de las personas autistas. Por esta razón, el gobierno peruano a través del Ministerio del Interior, otorgó el 2 de abril de 2020 (en el marco del día mundial de la concientización sobre el autismo) la posibilidad de salidas terapéuticas diarias de 15 minutos para personas autistas con un acompañante ante la situación de ansiedad, depresión e incluso autoagresiones que se detectó en esta población.

Pero así como esta cuarentena generó este tipo de dificultades, mostró, también, que un gran número de autistas mostraron más facilidades para adaptarse mejor a esta nueva situación en comparación con la población no autista. El uso de mecanismos y plataformas de comunicación pareció prolongar una realidad comunicativa que les era preferente. ¿Cómo podemos entender este tipo de desempeño de la mente autista en el uso de tecnología en esta "nueva normalidad"? ¿Hay alguna relación entre este tipo de mente y el desarrollo de estas tecnologías de la información? Para tratar de responder estas preguntas se debe pensar sobre la etapa en que vivimos y que hemos dado en denominar como "electronalidad" y que aparece por primera como concepto en la historiografía (D’Haenens, 1983) y, luego, en la semiótica (Biondi y Zapata, 2006).

Sin embargo, fue Michael Foucault (1967) quizá el primero que señaló no sólo el advenimiento de nuestro mundo electronal sino, también, quien mostró su configuración: "Nadie ignora que la gran obsesión del siglo XIX, su idea fija, fue la historia: ya como desarrollo y fin, crisis y ciclo, acumulación del pasado, sobrecarga de muertos o enfriamiento amenazante del mundo. El siglo 
XIX encontró en el segundo principio de la termodinámica el grueso de sus recursos mitológicos. Nuestra época sería más bien la época del espacio. Vivimos en el tiempo de la simultaneidad, de la yuxtaposición, de la proximidad y la distancia, de la contigüidad, de la dispersión. Vivimos en un tiempo en que el mundo se experimenta menos como vida que se desarrolla a través del tiempo que como una red que comunica puntos y enreda su malla. Podría decirse acaso que las disputas ideológicas que animan las polémicas actuales se verifican entre los descendientes devotos". (Foucault, 1967/1984).

La electronalidad (Reaño, 2017), se refiere al tiempo que se abre con la incorporación de la palabra electrónica a nuestra producción comunicacional y de sentido. Nacida de las tecnologías de la cibernética e impulsada como orden semiótico con el arribo de la internet, desplazó la forma cómo concebíamos el mundo y otorgábamos sentido en la etapa escribal, aquella que desde la invención de la imprenta había colocado al libro y a la palabra escrita como aquella que privilegiaba nuestra manera de objetivar la realidad.

En la última década se ha avanzado en la idea de que esta etapa del desarrollo semiótico humano es producto de la mente autista (Reaño, 2017). El cerebro autista tiene una gran capacidad de sistematizar información, unido a habilidades de hiperfocalización en temas de interés y almacenamiento en la memoria a largo plazo. Estos tipos de cerebros han sido claves en muchas etapas de generación de tecnología a lo largo de la historia de la humanidad (Armstrong, 2010). En la actualidad, podemos constatar que siendo el autismo el $1 \%$ de la población, esta distribución aumenta significativamente en lugares donde se produce tecnología informática (Roelfsema et al., 2012; Silberman, 2001), en aquellos enclaves donde se necesitan, sobre todo, programadores, ingenieros $y$ operarios para el desarrollo y diseño de software, hardware y aplicaciones digitales. Cuando analizamos las características de de las funciones de la electronalidad con la manera cómo procesan el sentido las personas autistas, encontramos semejanzas notables. $Y$ diferencias en la manera cómo se producía el sentido en la etapa escribal, es decir, en aquella donde se privilegiaba la palabra escrita y el libro impreso era el depositario del conocimiento.

\section{Segunda función de la electronalidad y producción del sentido en el autismo}

Existen tres funciones que explican la manera cómo la humanidad ha privilegiado la producción del sentido de acuerdo con las tecnologías de la comunicación humana utilizadas a través de 3 grandes etapas: la oral, la escribal y la electronal (Reaño, 2017):

Función 1: Realidad objetiva vs. realidad subjetiva.

Función 2: Privilegio del diálogo vs. privilegio del dialogismo.

Función 3: Privilegio de la lógica intensional vs. privilegio de la lógica extensional.

La realidad puede ser una serie de eventos objetivados en contraposición con la perspectiva (subjetiva) que podamos tener de ellos. Esto era notorio en la etapa escribal. La manera cómo se privilegiaba el sentido en ella hacía que la verdad se considerara objetiva frente al conocimiento que se hallaba en los libros. Los axiomas matemáticos, por ejemplo, son una muestra de objetivación de la realidad donde uno no puede tener una opinión. De similar modo, sobre ciertas definiciones, ideas y datos que se encontraban en textos escritos no solían ser pensados como falsos por el hecho de encontrarse en un "libro", prefigurado como el depositario del saber y de nuestra herencia acumulativa cultural. 
En nuestra etapa electronal, la expresión de la subjetividad es una de las características más notorias del espíritu de de los tiempos actuales. Las redes sociales, por ejemplo, son expresión de opiniones que, por el simple hecho de ser expresadas, son consideradas sino como verdaderas, poderosamente como válidas por el usuario que las emite. La irrupción de las "fake news", es un ejemplo de la pérdida de límites y parámetros objetivos con los cuales analizar la información, generar opiniones sobre política o económica apelando a la sola subjetividad es usual en nuestro tiempo. No es mi intención, como lingüista, calificar esta situación sino apuntar a hechos comprobables en el uso que se le da a la palabra electrónica. Esta misma democratiza, también, las posibilidades de expresión y de validación de colectivos neurodivergentes que tenían una muy reducida posibilidad de enunciarse en la etapa escribal. Antes de la aparición de la internet, hubiese sido poco probable que una persona autista publicase un libro o supiésemos de sus vivencias, para una persona autista no hablante que se valiera de un sistema de comunicación alternativa habría sido imposible. Hoy, conocemos cientos de testimonios y relatos de estas poblaciones. En la electronalidad, se da la figura del "prosumidor", es decir, del que puede crear contenidos y no sólo consumirlos, gracias al acceso a herramientas informáticas y al espacio libre, en muchos casos, que significa la red digital mundial.

En el autismo, la mediación de una atención compartida, la emergencia de sus formas empáticas, está dada por sus intereses profundos. La manera cómo se aproxima la persona autista a los objetos y saberes del mundo es altamente subjetiva y dependerá de sus campos de interés, en los cuales suelen convertirse en verdaderos expertos del tipo de sistema que elijan privilegiar. Durante mucho tiempo se calificó a estos intereses como "restringidos" y ligados a una obsesividad y la inflexibilidad. Sabemos, gracias a relatos de personas autistas que se trata, más bien, en un sentido lejano a la psicopatología, de "intereses profundos" (Shore, 2012), a los que se accede gracias a la pasión por el detalle en las mentes autistas.

La segunda función de la electronalidad plantea el privilegio de la conectividad sobre el dialogismo. Dentro de nuestras formas comunicativas que elegimos privilegiar: el diálogo que se dirige a un otro y espera una respuesta en la inmediatez o la conectividad que sabe de distancia y para la cual basta que el otro esté, presente o ausente, con una respuesta que puede ser diferida, no llegar como directaauna demanda decomunicación. La electronalidad puede ser vista como una "hiperculturalidad", siguiendo a Byung-Chul Han (2005/2019), es decir, una construcción de espacios culturales que no tiene centros y donde se privilegia la cercanía, la yuxtaposición, la mezcla, en un universo de interconexiones digitales que hace que podamos estar en muchos y ningún lugar a la vez. Este es el sentido de la gran red que presagiaba Foucault (1967/1994) en el texto citado anteriormente.

La electronalidad, a través de la aparición de las charlas virtuales ("chat"), ha logrado un efecto de abrir una ventana al diálogo que supera la distancia, pero también al privilegio del estar conectado. Se pudden tener varias conversaciones al mismo tiempo donde algunas serán prioritarias y otras se darán por el hecho de señalar un "aquí estoy" que enganche una cercanía que no busque una respuesta inmediata más que la certeza de la posibilidad de una interacción futura; se privilegia, entonces, el sabernos conectados antes que el diálogo inmediato. Los detalles se superponen al todo, la posibilidad a la efectividad. 
En el autismo encontramos un tipo de "pensamiento en detalles": se procesa preferentemente de las partes al todo, de manera inversa al funcionamiento típico. Este tipo de funcionamiento, unido al establecimiento de interacción sobre la base de intereses profundos, hace que la persona autista busque más el estar "conectado" con los demás de manera intermitente que sostenida. La fluidez se establecerá prioritariamente al aparecer un tema afín a sus intereses sistemáticos.

\section{La palabra electronal y la conectividad: el caso del WhatsApp}

La electronalidad bosqueja un sistema cultural donde se puede reflexionar nuevamente en la idea de Benveniste (2012) a propósito de la palabra electrónica: "En todo tiempo y lugar la escritura fue el instrumento que permitió a la lengua semiotizarse a sí misma". Es decir, de la función instrumental de la lengua se pasa a una representativa. ¿Qué pasa, entonces, en nuestros días con la escritura del texteo, de los 280 caracteres en Twitter, pero sobre todo, de los emojis?

Los emojis representan acaso un tipo nuevo de escritura en ciernes, pictográfica (eso significa, literalmente, 'emoji' en japonés: 'pictografía') que buscaría iconizar los referentes del mundo, expresar directamente conceptos sin tener que recurrir a la grafía, buscando determinada economía situacional generada, sobre todo, por las redes sociales, que incluyen chats y para las que los emojis dejan de ser simples marcas paralingüísticas (acompañantes que reemplazan la emocionalidad asociada al enunciado cara a cara; en esto vemos, también, otra marca del retorno de la palabra electronal a la palabra hablada) para convertirse, por momentos, en el texto mismo.

No es inusual que, como hemos visto, al "textear" en WhatsApp respondiendo a un amigo que nos pregunta si, de todas maneras, nos veremos hoy como habíamos quedado, pero a nosotros nos ha surgido un imprevisto $X$ y no podremos asistir, así que en la pantalla ponemos el siguiente texto: he tenido un problema, no voy a poder ir.

En ese momento, antes de enviar el mensaje, por un momento juzgamos que "suena" demasiado duro y decidimos darle un énfasis sobre nuestra emoción o sobre lo que debería de entenderse por ella:

He tenido un problema, no voy a poder ir 6

Figura 1. Vista en pantalla del uso del Emoji para hacer énfasis en la emoción

Con lo cual añadimos los elementos extralingüísticos faltantes: la entonación y el gesto que la frase por sí sola no contenía. En muchas ocasiones al enviar un mensaje por este medio no esperamos una respuesta inmediata. Nos basta saber que en algún momento se nos leerá y responderá. Es la idea de estar conectados la que nos liga con el otro, como si existiese una comunicación permanente que en su virtualidad pudiese actualizarse en cualquier instante.

Y es que el diálogo como tal va quedando fuera 
no sólo de las preferencias del usuario sino de su estilo comunicativo, marca del tiempo de la electronalidad. Así, por ejemplo, los mensajes de audio en WhatsApp hacen que podamos tener una comunicación donde el término de un audio posibilite, o no, una respuesta:

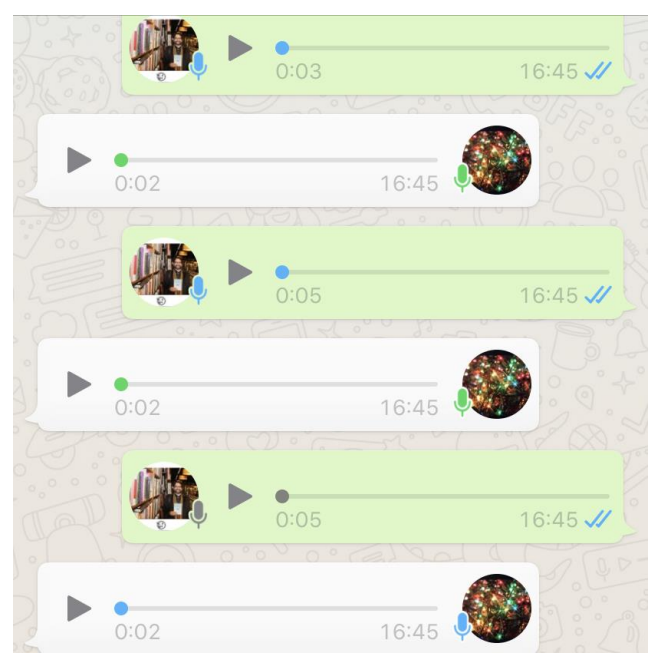

Figura 2. Mensajes de audio por WhatsApp

En la figura 2, quizá podríamos preguntarnos si no hubiese sido más fácil llamar por teléfono al interlocutor. La razón es que queremos comunicarnos pero no bajo una interacción que se base en un esquema dialógico, que involucre una pregunta-respuesta en el instante, deseamos estar, sí, conectados, disponer de la tranquilidad y predictibilidad de crear nuestro propio mensaje fuera de la escucha instantánea del otro.

Para el tipo de comunicación en el autismo, los emojis y mensajes de audio, son herramientas que facilitan su interacción. Los emojis, en cuanto expresan emociones, son altamente predecibles porque como elemento paraverbal no varían como lo hace el rostro humano (sin dejar de tener en cuenta que la combinación de emojis puede llevar a diferentes sentidos) y facilitan su comprensión y expresión por parte del usuario autista que utiliza la palabra electronal.
El mensaje de audio, minimiza también la ansiedad que una comunicación directa puede ocasionar en estas personas; es, además, predecible: puedo darme el tiempo para meditar mi respuesta, modificarla o incluso borrarla.

Cabe plantearse por qué una herramienta de comunicación que favorece a las personas autistas, que está ligada a su estilo de procesamiento cognitivo, es tan popular y le permite a la población neurotípica encontrarle similares beneficios, es decir, el privilegio del poder estar conectados sin buscar, principalmente, la inmediatez de la respuesta que otorga el diálogo. El estar "conectado" pareciera ser un corolario de adaptación a estas nuevas herramientas creadas en nuestra electronalidad, una forma de poder fluir en este mundo hiperconectado sin la sobrecarga que supondría, en la población típica, el mantener tantos diálogos sin perder el hilo de múltiples conversaciones virtuales. 
El avance de la comprensión sobre el autismo revela la gran capacidad de estas personas para tareas que requieren un elevado grado de sistematización y la concentración de este tipo de talento en zonas donde se produce tecnología informática. De la creación de herramientas que superan las dificultades que ellos encuentran en la comunicación, nos encontramos que sus beneficios pueden ser importantes para la población típica también.El modo de comunicación actual (acrecentado por el aislamiento físico producto de la pandemia) se torna cada vez más autista porque esta era está creada por un tipo de cerebros que se encuentra en esta forma de neurodivergencia. $Y$ así como en otras áreas, es el inicio de los beneficios que empezamos a reconocer en el uso de tecnologías que, a su base, están pensadas para favorecer la diversidad. Conocer el funcionamiento de otro tipo de mentes puede inspirar y darnos claves para nuestras relaciones y manejo de las habilidades sociales en esta "nueva normalidad".

\section{REFERENCIAS BIBLIOGRÁFICAS}

Benveniste, É. (2012). Dernières leçons. Collège de France 1968 et 1969. Paris: Éditions du Seuil/Gallimard.

Biondi J. y Zapata, E (2006) La palabra permanente. Lima: Fondo Editorial del Congreso del Perú.
Byung-Chul Han (2005/2019). Hiperculturalidad. Barcelona: Herder.

D’Haenens, A. (1983) Oralité, Scribalité, Electronalité: La scribalité occidental depuis le moyen Âge. Louvain-la-Neuve.

Foucault, M. Des espaces autres " (conférence au Cercle d'études architecturales, 14 mars 1967), Architecture, Mouvement, Continuité, $n$ “5, octobre 1984, pp. 46-49.

Reaño, E. (2017). El retorno a la aldea. Neurodiversidad, autismo y electronalidad. Lima: Perro de Ambiente Editor.

Roelfsema, M. T. et al (2012) Are Autism Spectrum Conditions More Prevalent

in an Information-Technology Region? A School-Based Study of Three Regions in the Netherlands. J Autism Dev Disord (2012) 42.

Shore, S. (2012). A different order of being. En: Santomauro, J. (Ed.) Autism all stars. Londres: Jessica Kingsley Publishers.

Silberman, S. (2001). The geek syndrome. Recuperado el 28/7/2020: https://www.wired. com/2001/12/aspergers/

Simon Baron-Cohen (2008). Autism and Asperger Syndrome. Oxford: Orford University Press. 